Paediatr.Paedolog. 2020 · 55 (Suppl 3):S120-S124 https://doi.org/10.1007/s00608-020-00808-y

c Der/die Autor(en) 2020

\section{R. Mair ${ }^{1} \cdot$ G. Tulzer ${ }^{2}$}

' Department für Kinderherzchirurgie, Klinik für Herz-Thorax Chirurgie, Kepleruniklinikum Linz, Linz, Österreich

${ }^{2}$ Klinik für Kinderkardiologie, Kepleruniklinikum Linz, Linz, Österreich

\title{
Kinderherzchirurgie: am Beispiel des Kinderherzzentrums Linz
}

\section{Hintergrund}

\section{Gerald Tulzer}

Bis 1995 gab es in Österreich an den 3 medizinischen Fakultäten (Wien, Graz und Innsbruck) eigene Abteilungen für Kinderkardiologie. Die angeborenen Herzfehler im Kindes- und Jugendalter wurden an den dortigen Herzchirurgien operiert. Bis 1995 mussten Kinder aus Österreich immer wieder ins Ausland geschickt werden, da komplexere Korrekturen angeborener Herzfehler an Neugeborenen kaum durchgeführt wurden. Manche Herzfehler, unter anderem das hypoplastische Linksherzsyndrom wurden gar nicht angegangen, Fontan-Operationen nur in ausgewählten Fällen selten durchgeführt und eine postnatale Comfort Care (Verzicht auf jede Therapie) war durchaus üblich.

An der damaligen Landes-Frauenund Kinderklinik gab es primär keine Kardiologie, aber eine bedeutende Anzahl an herzkranken Neugeborenen und Kindern. Im Jahr 1987 begann ich auf Eigeninitiative eine Ausbildung in Echokardiographie (die Basis für den Aufbau einer kardiologischen Ambulanz bzw. eines kardiologischen Programms). Von 1989 bis 1990 konnte ich dann ein Fellowship am Children's Hospital of Philadelphia absolvieren unter der damaligen Führung von Prof. Dr. J. C. Huhta (Kardiologie) und Prof. Dr. W. Norwood (Herzchirurgie). Nach der Rückkehr nach Linz wurde mit dem Aufbau des kinderkardiologischen Programms begonnen: Herzambulanz, fetale Echokardiographien, Betreuung kardiologischer stationärer Patienten, einfache Herzoperationen etc. Aufgrund der rasch steigenden Patientenzahlen wurde dann vom damaligen ärztlichen Direktor Prim. Dr. Wilhelm Tulzer bereits 1993 ein eigenes Department für Kinderkardiologie gegründet. Parallel mit dem Aufbau dieser Strukturen wurde auch Kontakt mit Herzchirurgen gesucht, die sich auf Kinderherzchirurgie spezialisieren möchten, und mit Dr. Rudolf Mair wurde auch ein kongenialer Partner gefunden.

\section{Beginn und Entwicklung der Kinderherzchirurgie in Linz}

\section{Rudolf Mair}

In Linz bestand eine sehr aktive Herzchirurgie, die jedoch ausschließlich auf Behandlung erworbener Herzerkrankungen ausgerichtet war. Um ein Programm zur chirurgischen Behandlung angeborener Herzfehler zu beginnen, musste jemand auf diesem Gebiet ausgebildet werden. Um das Gelernte wirklich sinnvoll anwenden zu können und die erforderliche Qualität zu garantieren, braucht es eine gewisse Mindestanzahl an Fällen. Ferner sollten sich langfristig mindestens 2, besser 3 Chirurgen mit diesem Gebiet identifizieren. Dies ergibt dann automatisch eine gewisse Mindestgröße eines solchen Programms und gewährleistet eine konstant verfügbare Qualität.

Österreich hatte 1994 etwa 7.500.000 Einwohner und bei etwa 70.000 Geburten einen Bedarf von etwa 600 Operationen jährlich. Nachdem insbesondere von den komplexeren Fällen viele Kinder ins Ausland geschickt werden mussten, war nur ein qualitativ sehr hochwertiges Zentrum, das keine Kinder ins Ausland verschicken muss, sinnvoll.

Ich war damals bereits Facharzt für Chirurgie und kurz vor dem Abschluss meiner Zusatzausbildung zum Facharzt für Herzchirurgie. Dank der Offenheit und des Weitblicks von Prof. Dr. Aldo Castaneda Leiter der Herzchirurgie des Children's Hospital Boston, gelang es mir, dort ein Clinical Fellowship (Senior Resident) für eine Rotation (6 Monate) vom 01.07.1994 bis 31.12.1994 zu bekommen.

Unterstützt wurde das ganze Projekt vom Leiter der Abteilung für Herz-, Thorax- und Gefäßchirurgie am $\mathrm{AKH}$ Linz Prof. Dr. P. Brücke und dem damals interimistischen ärztlichen Leiter des Linzer Allgemeinen Krankenhauses Prof. Dr. D. Klingler. Es gelang auch noch, für unsere Kollegin von der Abteilung Anästhesie und Intensivmedizin, Gertaud Geiselseder, einen Ausbildungsaufenthalt an der Cardiac ICU sowie an der Abteilung für Anästhesie des Children's Hospital Boston zu bekommen. Neben der hervorragenden klinischen Ausbildung konnte man dort auch sehr viel hinsichtlich Organisation und Führungsverhalten lernen. Es gab zwar Abteilungen für kardiovaskuläre Chirurgie, Kardiologie und Anästhesie; die Kooperation war aber sehr eng. Es gab eine Bettenstation, auf der sämtliche kardiologischen und herzchirurgischen Patienten gemeinsam betreut wurden. Es gab eine kardiologische Intensivstation, auf der ebenfalls herzchirurgische und kardiologische Patienten betreut wurden. Zum Intensivteam gehörten pädiatrische Intensivmediziner (Kar- 


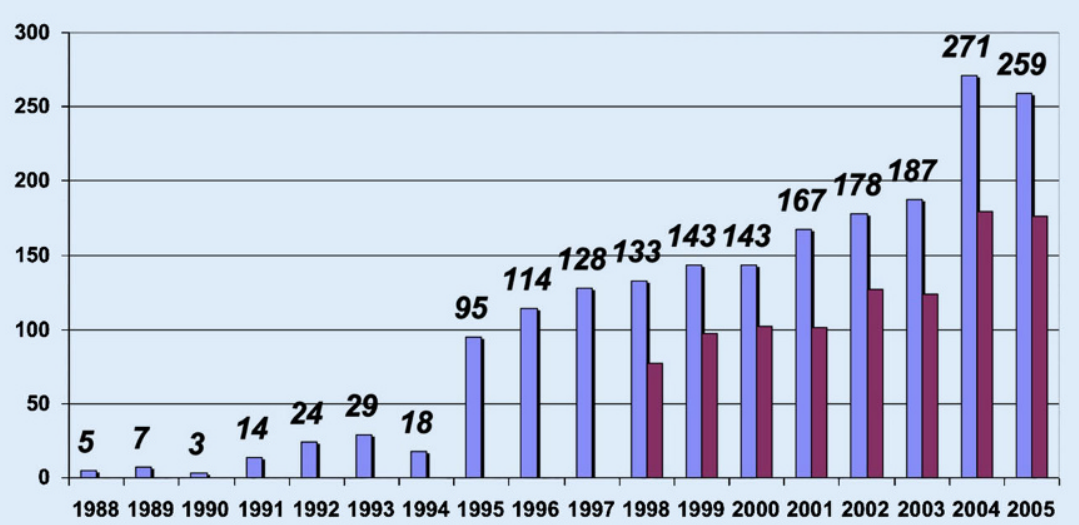

Abb. 1 - Diese Abbildung zeigt den Verlauf der Anzahl an Kinderherzoperationen am Kinderherzzentrum Linz von 1988 bis 2005. Die dunkelroten Säulen repräsentieren die OPs an Kindern unter 1 Jahr (KUK, Klinik für Kinderkardiologie)

diologen und Anästhesisten), Residents beider Fachrichtungen und chirurgische Ausbildungsassistenten. Diese enge Kooperation und Verflochtenheit der Fachdisziplinen war für uns das organisatorische Modell, das es bei uns möglichst 1:1 umzusetzen galt. Es war uns durchaus bewusst, dass so etwas im dortigen System, in dem eine wesentlich flachere hierarchische Pyramide besteht, leichter umzusetzen ist, als in unserem von Territorialitätsverhalten geprägten Spitalswesen.

Für ein funktionierendes tertiäres Zentrum sind zusätzlich eine spezialisierte Anästhesie und eine spezialisierte Intensivmedizin erforderlich. Um den Anforderungen einer modernen Kinderkardiologie gerecht $\mathrm{zu}$ werden, bedarf es überdies eines gut funktionierenden Programms in fetaler bzw. pränataler Medizin.

\section{Umsetzung und Start des Kinderherzzentrums Linz}

Mit der Rückkehr aus Boston Mitte Januar 1995 begannen wir unser Kinderherzprogramm sowohl medizinisch als auch strategisch zu entwickeln.

Strategische und organisatorische Voraussetzungen:

Das bereits als eigenständige organisatorische Einheit existierende Department für Kinderkardiologie war Teil der Landes-Kinderklinik. Träger war das Land Oberösterreich. Herzchirurgie und die für herzchirurgische Anästhesie und
Intensivmedizin zuständige Anästhesieabteilung befanden sich im Allgemeinen Krankenhaus. Träger war die Stadt Linz. Geographisch waren die beiden Häuser unmittelbar benachbart und nur durch die Krankenhausstraße getrennt. Ein neues Herzkatheterprogramm für Kinder mit angeborenen Herzfehlern wurde initiiert. Das Herzkatheterlabor im Allgemeinen Krankenhaus wurde für Kinder an 2 Tagen pro Woche angemietet.

Unterstützt wurde die Umsetzung vom Leiter der Herzchirurgie Prof. Dr. P. Brücke sowie vom ärztlichen Leiter des Allgemeinen Krankenhauses Prof. Dr. G. Syre. Aufseiten der Anästhesie und Intensivmedizin unterstützte uns Doz. Dr. St. Necek, auf der Seite der Kinderklinik Prof. Dr. K. Schmitt. Es wurde uns ermöglicht, uns zu spezialisieren und eigene Mannschaften zu formen. Auch von den Spitalsträgern, der Stadt Linz und dem Land Oberösterreich kam trotz des unvorteilhaften auf Defizitabdeckung basierenden Spitalsfinanzierungssystems in der Person des zuständigen Stadtrats DI Erich Haider klarer Support. Ebenso vorbehaltlos unterstützt wurde unser Vorhaben vom Land Oberösterreich, allen voran vom damaligen LH Dr. Josef Pühringer.

\section{Medizinischer Bereich}

In der direkten Arbeit am Patienten war die Umsetzung vollkommen klar. Es gab außer uns in den beteiligten Bereichen niemanden, der über entsprechende Er- fahrungen verfügte, sodass wir von Anfang an medizinisch völlig eigenständig und unabhängig entscheiden konnten. Nur in interdisziplinären Einrichtungen wie der Intensivmedizin bedurfte es einiger Zeit, um Kompetenzfragen und Verantwortlichkeiten zu klären.

Alle Patienten wurden im Team besprochen. Die präoperative Betreuung erfolgte durch Kardiologen auf der kardiologischen Bettenstation oder, wenn der Patient intensivpflichtig war, auf der entsprechenden Intensivstation (neonatale Intensivstation oder pädiatrische Intensivstation). Operiert wurde im Operationssaal der Herzchirurgie. Die postoperative Betreuung erfolgte primär auf der operativen Intensivstation durch Anästhesisten unter Einbeziehung von Kinderkardiologen und Kinderherzchirurgen. Im weiteren Verlauf wurden die Patienten wieder auf die kardiologische Station verlegt, wo sie von Kinderkardiologen und Kinderherzchirurgen betreut wurden. Aufgrund dieser sehr engen Kooperation bedurfte es, abgesehen von den Verlegungsberichten zwischen Intensiv- und Normalstation, keiner weiteren Arztbriefe zwischen den einzelnen Einrichtungen, da die handelnden ärztlichen Personen während des gesamten Behandlungsverlaufs die gleichen waren.

Es wurde von Anfang an nach modernsten Prinzipien und Erkenntnissen vorgegangen. Das Prinzip der frühen Vollkorrektur wurde uneingeschränkt umgesetzt. Palliative Eingriffe, die damals in Europa bei vielen biventrikulären Vitien weit verbreitet waren, waren bei uns ausschließlich auf Patienten mit univentrikulärer Physiologie beschränkt. Bezüglich der Komplexität von Herzfehlern setzten wir uns nach oben keine Grenzen. Bereits 1995 wurden unter anderem der Truncus arteriosus communis, die Taussig-Bing-Anomalie und selbstverständlich die Transposition der großen Arterien im Neugeborenenalter primär vollkorrigiert. Bereits 1997 konnten wir erfolgreich einige Patienten mit hypoplastischem Linksherzsyndrom behandeln. Bereits im Jahr 2000 wurde bei kritischer Aortenklappenstenose die Ross-Konno-Operation im Neugeborenenalter erfolgreich durchgeführt. Dadurch konnten wir die häufiger wer- 
denden Fälle dieses Vitiums, die durch unser erfolgreiches fetales Interventionsprogramm zu uns kamen, entsprechend biventrikulär versorgen. Unsere Rate an sehr guten biventrikulären Resultaten nach fetaler Intervention war damit wesentlich höher als in anderen Spitzenzentren. Im Jahr 2003 wurde bei uns erstmals eine En-bloc-Rotation der Ausflusstrakte bei Transposition der großen Arterien mit Ventrikelseptumdefekt und linksventrikulärer Ausflusstraktobstruktion durchgeführt. Damit gelingt es, dieses Vitium vollständig anatomisch mit Wachstumspotenzial in allen tubulären Strukturen $\mathrm{zu}$ rekonstruieren. Ab dem Jahr 2000 konnten wir in Kooperation mit unserer Pränatalmedizin als erstes europäisches Zentrum ein Programm für pränatale kardiale Interventionen starten, das heute das größte in Europa ist.

Von anfangs 96 Operationen im Jahr 1995 kam es zu einer ständigen Zunahme der Fälle, sodass wir im Jahr 2006 bereits mehr als 300 Fälle operierten (• Abb. 1). Seither bewegen wir uns bei Fallzahlen zwischen 300 und 350 pro Jahr. Parallel dazu wurden unsere Operationskapazitäten erhöht von anfangs 2 Operationstagen pro Woche aufheute 7 Operationssäle pro Woche.

Voraussetzung für all das waren sehr gute Ergebnisse, die wir bereits ab 1996 vollständig in einer eigenen Datenbank dokumentierten.

Im Jahr 2003 wurde durch die Publikation eines Buchs von Waldhauser, Jürgensen und Püspök (Weggelegt; • Abb. 2) das Thema Qualität in der Kindermedizin aufgearbeitet. Auch der Kinderherzchirurgie war ein großes Kapitel gewidmet. Das hat letztendlich auch zu einem externen Audit von Prof. Castaneda geführt, das dann die Bedeutung von Zentren für die Behandlungsqualität hervorgehoben hat.

Im Jahr 2013 wurde die eigene Datenbank durch die vollständige Teilnahme an der Datenbank der European Congenital Heart Surgeons Association ersetzt (• Abb. 3). Dies hatte in einigen Bereichen interessante Auswirkungen: So sank beispielsweise die Mortalität der RossKonno-Operation im Neugeborenenalter in der gemeinsamen europäischen

Paediatr. Paedolog. 2020 · 55 (Suppl 3):S120-S124 https://doi.org/10.1007/s00608-020-00808-y (c) Der/die Autor(en) 2020

\section{R. Mair · G. Tulzer}

\section{Kinderherzchirurgie: am Beispiel des Kinderherzzentrums Linz}

\section{Zusammenfassung}

Die Entstehung und Weiterentwicklung des Kinderherzzentrums Linz entsprang unserer eigenen Initiative und der Erkenntnis, dass enge interdisziplinäre Zusammenarbeit von größter Bedeutung ist. Sie wurde uns nicht verordnet, sie wurde uns aber durch weitblickende Abteilungsvorstände und Politiker ermöglicht. Voraussetzungen waren hohe fachliche Kompetenz aller Beteiligten, ausgeprägte Teamfähigkeit und Kommunikationsfähigkeit sowie der Wille zu Veränderung.

Die sehr positive Weiterentwicklung unseres Zentrums sowie die schrittweise Zentralisierung sind primär das Resultat außerordentlich guter klinischer Ergebnisse, aber auch eines einigermaßen leistungsfreundlichen Umfelds aus Spitalsmanagement und lokaler Gesundheitspolitik. Das österreichische Spitalswesen ist trotz seiner Verdienste aufgrund seines administrativen Systems (Finanzierung, Personalzuteilung etc.), das sich im Wesentlichen an Belagstagen, Bettenanzahl, Postleitzahlen und ähnlichem orientiert, für Innovationen, Leistungssteigerung, Qualitätsverbesserung und Effizienzsteigerung sehr schwerfällig. Eine leistungs- und kompetenzgerechte Honorierung sowohl des Zentrums als auch seiner Mitarbeiter ist dringend erforderlich.

\section{Schlüsselwörter}

Kinderherzchirurgie - Kinderkardiologie . Kinderherzzentrum - Behandlungsqualität · Interdisziplinäre Zusammenarbeit

\section{Cardiac Surgery in Children: Example of the Children's Heart Centre, Linz}

\section{Abstract}

The emergence and further development of the Children's Heart Center in Linz came from our own initiative and the realization that close interdisciplinary cooperation is of utmost importance. It was not prescribed to us, but it was made possible by far-sighted department heads and politicians. The prerequisites were a high level of professional competence of all those involved, a strong ability to work in a team and to communicate, as well as the will to change.

The very positive development of our center and the gradual centralization are primarily the result of extraordinarily good clinical results, but also a reasonably performancefriendly environment consisting of hospital management and local health policy. Despite its merits, the Austrian hospital system is very rigid concerning innovations, performance enhancement, quality improvement and efficiency increase owing to its administrative system (financing, personnel allocation, etc.), which is mainly based on the number of days of occupancy, number of beds, postcodes, and the like. Rewarding both the center and its employees in line with performance and competence is urgently required.

\section{Keywords}

Pediatric cardiac surgery - Pediatric cardiology - Children's Heart Center - Quality of care . Interdisciplinary cooperation
Datenbank ab 2013 durch unsere Teilnahme deutlich.

\section{Strategische Umsetzung}

Voraussetzung für jede strategische Weiterentwicklung war die sofortige und saubere Umsetzung unserer Kenntnisse im medizinischen Bereich, die zu außergewöhnlich guten Ergebnissen führte. Auf dieser Basis konnten und mussten wir sehr rasch strategische Schritte setzen.

Die einzige eigenständige organisatorische Einheit in unserem Bereich war das
Department für Kinderkardiologie. Dieses verfügte über eine geringe Anzahl von Betten und über eine kleine Ambulanz. Chirurgisch, anästhesiologisch und intensivmedizinisch gab es keine, von ihren Stammabteilungen organisatorisch abgegrenzte Einheiten. Der Betrieb des Kinderherzprogramms war also vom Wohlwollen der jeweiligen Abteilungsvorstände abhängig. Strategisches Ziel war daher klarerweise eine komplette fachliche und größtmögliche organisatorische Unabhängigkeit, so sehr wir den Mutter- 


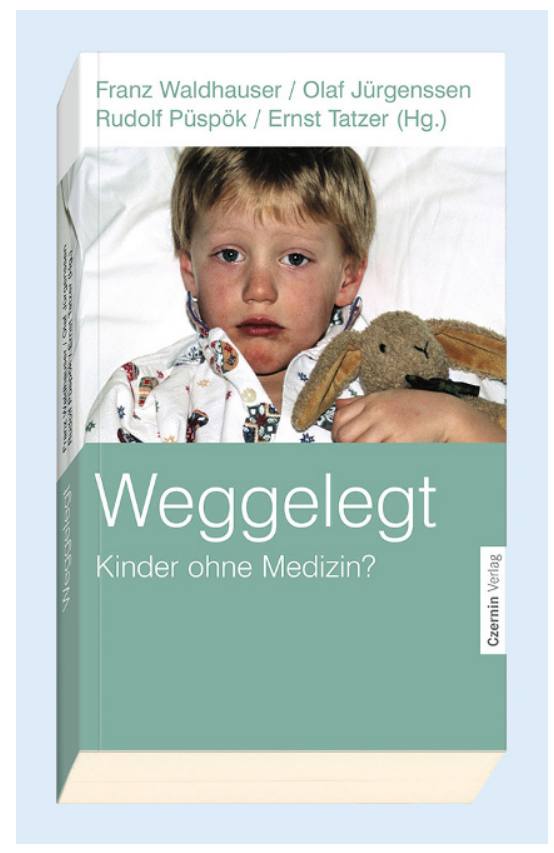

Abb. $2 \Delta$ Cover des Buchs Weggelegt $(\odot$ Czernin Verlag)

abteilungen und den fördernden Abteilungsvorständen verbunden waren.

Von Beginn an wurde Interdisziplinarität, die wir während unserer Spezialausbildung erlebt hatten und im medizinischen Bereich sofort umsetzten, auch in unserem Auftreten nach außen getragen.

Der gemeinsame Auftritt von Kardiologie, Kinderherzchirurgie, Anästhesie und Intensivmedizin nach außen wurde sofort Standard. Es wurde ein Logo besorgt und wir nannten uns von Anfang an „Kinderherzzentrum Linz“. Der Begriff „Kinderherzzentrum“ wurde von uns geprägt. Dies alles geschah in Eigeninitiative, noch vor den ersten gemeinsamen offiziellen administrativen Schritten beider Spitalsträger. Ebenso wurde sofort mit dem Beginn des kinderherzchirurgischen Programms auch mit der Anwerbung und Ausbildung junger Kolleginnen und Kollegen begonnen.

In einem Spitalswesen, in dem das ärztliche Einkommen zu einem wesentlichen Teil aus Zusatzeinkünften (Sonderklassehonorare, Nacht-, Sonn- und Feiertagszulagen etc.) besteht, ist es schwierig, junge Leute für ein Programm zu motivieren, in dem diese Zusatzeinkünfte größtenteils wegfallen.

Wir konnten aber kompensatorisch einiges anbieten:

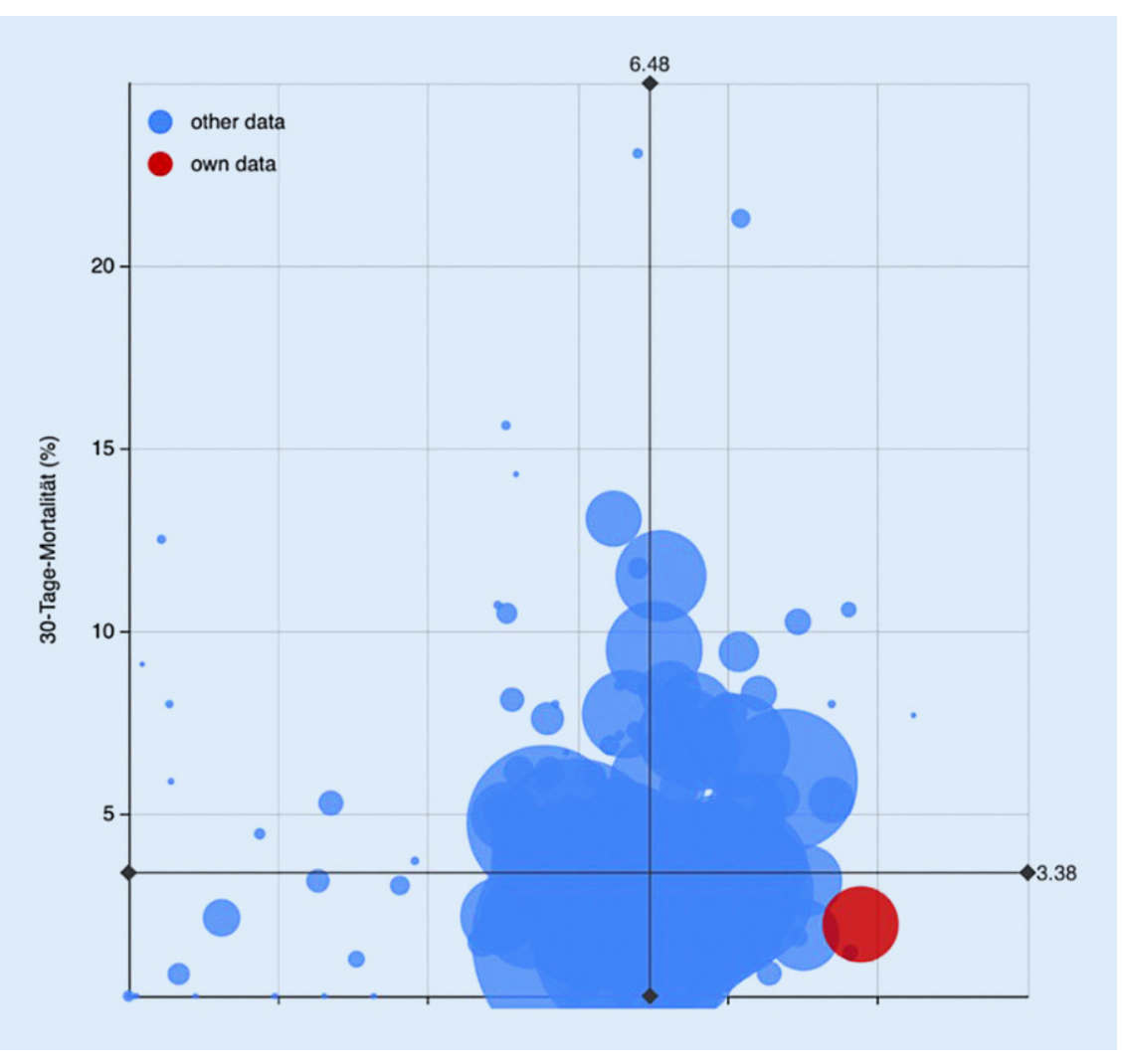

Abb. $3 \Delta$ Quality of Care Chart ECHSA Congenital Database. 30-Tage-Mortalität bei Kinderherzoperationen einzelner Zentren ( $y$-Achse) in Relation zur Komplexität des Herzfehlers ( $x$-Achse) anhand des Aristoteles Scores ( 0 = einfache Herz-OP, 10 = höchste Komplexität). Die Größe der Kreise zeigt das Volumen eines Zentrums an. Der Kreis in rot entspricht dem Kinderherzzentrum Linz (ECHSA Congenital database; https://www.echsacongenital.org/quc_report/ans)

- Exzellente Ausbildung

- Angenehmes Betriebsklima

- Umschriebenes, aber sehr vielfältiges und interessantes Fachgebiet

- Gewisse Exklusivität

- Gute fachliche Perspektive

- Eigenverantwortlichkeit

- Patientenbezogenes Arbeiten

- Keine Hausdienste als Facharzt etc.

Ab 2004 bekamen wir eine 2. Facharztstelle, die mit Frau Dr. E. Sames-Dolzer, die bei uns ausgebildet worden war, besetzt wurde. Im Jahr 2016 bekamen wir mit Frau Dr. M. Kreuzer, ebenfalls bei uns ausgebildet, eine 3. Fachärztin. Da beide Oberärztinnen Teilzeitstellen innehaben, sind wir einschließlich meiner Person zum jetzigen Zeitpunkt 2,75 Fachärzte. Zusätzlich sind noch 2 Assistenzärzte auf Vollzeitstellen in Ausbildung an unserem Department.

Aufgrund des zunehmenden Arbeitsaufwands in der Kinderherzchirurgie war es notwendig, unsere Gruppe schrittweise aus der Dienstmannschaft auszugliedern. Abgeschlossen war dieser Prozess 2003 mit der gleichzeitigen Einführung einer eigenen kinderherzchirurgischen Rufbereitschaft.

War Dr. Gerald Tulzer anfangs als Facharzt in der Kinderkardiologie allein, so wurden schrittweise neue Stellen geschaffen: mit der Schaffung des Departments, mit der Inbetriebnahme eines neuen eigenen Herzkatheterlabors, mit der Umwandlung in eine eigene Abteilung, sodass die Klinik für Kinderkardiologie heute neben dem Vorstand noch 8 Facharztstellen und 4 Assistentenstellen ausweist. Die Herzintensivstationen werden dienstlich von der Abteilung für Anästhesie und Intensivmedizin versorgt, eine Kinderkardiologin bzw. ein Kinderkardiologe ist immer in Bereitschaft.

Im Jahr 2003 kam es schließlich zu einem Kooperationsvertrag zwischen dem 
Land Oberösterreich und der Stadt Linz über Organisation und Finanzierung des Kinderherzzentrums.

Im Jahr 2009 konnte das Department für Kinderkardiologie schließlich in eine eigenständige Abteilung (Primariat) umgewandelt werden.

Mit April 2016 erlangte die kinderherzchirurgische Gruppe den Status eines eigenen Departments für Kinderherzchirurgie.

Die sehrkomplexe, sowohl abteilungsübergreifende, als auch trägerübergreifende Struktur und Arbeitsweise erforderte schließlich auch eine eigene Administration. Seit 2010 haben wir eine eigene administrative Kraft. Mittlerweile ist durch die zunehmende Dokumentation in allen Bereichen, die Erhöhung des Mitarbeiterstands, die hohen Fallzahlen und die Zunahme internationaler Patienten der administrative Aufwand so groß, dass zwei Teilzeitkräfte ihn eben noch bewältigen.

Ein weiteres Problem in der Umsetzung war die föderale Struktur unseres Gesundheitswesens in Verbindung mit dem sehr leistungsfeindlichen Finanzierungssystem. Um die entsprechende Fallzahl aufzubringen, braucht ein Kinderherzzentrum ein gewisses Einzugsgebiet. Man kann ein Kinderherzzentrum nicht bezirksweise aufbauen. Es genügt auch nicht bundesländerweise. Es braucht ein bundesländerübergreifendes Einzugsgebiet. Da die Spitalsfinanzierung aber Ländersache ist, erfordert es von den zuständigen Politikern die Bereitschaft, Geld für Patienten aus anderen Bundesländern auszugeben (sog. innerösterreichische Ausländer!?).

Die strategische Umsetzung ist ein sehr langwieriger Prozess. Es geht nur in sehr kleinen Schritten vorwärts und ist bis heute nicht abgeschlossen. Es braucht viel Überzeugungsarbeit, gute Argumente und Verantwortliche mit Weitblick.

\section{Korrespondenzadresse}

Prim. Priv. Doz. Dr. R. Mair
Department für Kinder-
herzchirurgie, Klinik für
Herz-Thorax Chirurgie,
Kepleruniklinikum Linz
Krankenhausstraße 26-30a,
4020 Linz, Österreich
Rudolfmair@
kepleruniklinikum.at

Prim. Univ.-Prof. Dr.
G. Tulzer
Klinik für Kinderkardiologie,
Kepleruniklinikum Linz
Krankenhausstraße 26-30a,
4020 Linz, Österreich
Gerald.tulzer@
kepleruniklinikum.at

Prim. Priv. Doz. Dr. R. Mair Promotion: 15. Juni 1982 Universität Wien September 1982 bis Juni 1983: Turnusarzt: Allgemeines Krankenhaus Linz Juli 1983 bis Dezember 1989: Facharztausbildung Chirurgie: I. Chirurgische Abteilung, Allgemeines Krankenhaus Linz Mai 1988 bis November 1988: Ausbildungsaufenthalt in Herzchirurgie: Klinik f. Herz-,Thorax- und Gefäßchirurgie, Medizinische Hochschule Hannover Januar 1990: Facharzt f. Chirurgie März 1993: Zusatzfacharzt für Gefäßchirurgie Juli bis Dezember 1994: Zusatzausbildung (Senior Resident) in Kinderherzchirurgie am Children's Hospital Boston März 1996: Zusatzfacharzt für Herzchirurgie Januar 1995: Gründung des Kinderherzzentrums Linz Juni 2011: Habilitation an der Med. Universität Graz (Priv. Dozent) April 2016: Leiter des Departments für Kinderherzchirurgie; Kepler Universitätsklinikum Linz.

Prim. Univ-Prof. Dr. G. Tulzer Promotion 1983 an de Universität Wien, 1983-86 Turnus mit Jus Practicand in Linz, Ausbildung zum Facharzt für Kinder-und Jugendheilkunde 1986-1990 in Linz und Philadelphia 1993 Leiter des Department für Kinderkardiologie an der Landes-Frauen- und Kinderklinik, $1994 \mathrm{Ha}$ bilitation an der Universität Wien, 1995 Gründung und Leitung des Linzer Kinderherzzentrums, 2008 Zusatzfächer Kinderkardiologie sowie Neonatologie und Intensivmedizin, 2009 Primarius der Abteilung für Kinderkardiologie, 2013 Erhalt des Berufstitels: Universitätsprofessor, seit 2014 Vorstand der Klinik für Kinderkardiologie am Kepleruniklinikum in Linz.

Funding. Open access funding provided by Kepler Universitätsklinikum Linz.

\section{Einhaltung ethischer Richtlinien}

Interessenkonflikt. R. Mair und G. Tulzer geben an, dass kein Interessenkonflikt besteht.

Für diesen Beitrag wurden von den Autoren keine Studien an Menschen oder Tieren durchgeführt. Für die aufgeführten Studien gelten die jeweils dort angegebenen ethischen Richtlinien.
Open Access. Dieser Artikel wird unter der Creative Commons Namensnennung 4.0 International Lizenz veröffentlicht, welche die Nutzung, Vervielfältigung, Bearbeitung, Verbreitung und Wiedergabe in jeglichem Medium und Format erlaubt, sofern Sie den/die ursprünglichen Autor(en) und die Quelle ordnungsgemäß nennen, einen Link zur Creative Commons Lizenz beifügen und angeben, ob Änderungen vorgenommen wurden.

Die in diesem Artikel enthaltenen Bilder und sonstiges Drittmaterial unterliegen ebenfalls der genannten Creative Commons Lizenz, sofern sich aus der Abbildungslegende nichts anderes ergibt. Sofern das betreffende Material nicht unter der genannten Creative Commons Lizenz steht und die betreffende Handlung nicht nach gesetzlichen Vorschriften erlaubt ist, ist für die oben aufgeführten Weiterverwendungen des Materials die Einwilligung des jeweiligen Rechteinhabers einzuholen.

Weitere Details zur Lizenz entnehmen Sie bitte der Lizenzinformation auf http://creativecommons.org/ licenses/by/4.0/deed.de.

Hinweis des Verlags. Der Verlag bleibt in Hinblick auf geografische Zuordnungen und Gebietsbezeichnungen in veröffentlichten Karten und Institutsadressen neutral. 